\title{
Lord Kelvin's method of images in semigroup theory
}

\author{
Adam Bobrowski
}

Received: 21 February 2010 / Accepted: 16 May 2010 / Published online: 8 June 2010

(C) The Author(s) 2010. This article is published with open access at Springerlink.com

\begin{abstract}
We show that Lord Kelvin's method of images is a way to prove generation theorems for semigroups of operators. To this end we exhibit three examples: a more direct semigroup-theoretic treatment of abstract delay differential equations, a new derivation of the form of the McKendrick semigroup, and a generation theorem for a semigroup describing kinase activity in the recent model of Kaźmierczak and Lipniacki (J. Theor. Biol. 259:291-296, 2009).
\end{abstract}

Keywords Abstract delay differential equations · Kinase activity · Signaling pathways $\cdot$ Bessel process $\cdot$ Boundary condition $\cdot$ McKendrick equation $\cdot$ Method of images

\section{The general idea}

Lord Kelvin's method of images is an ingenious way of solving problems involving boundary conditions, see e.g. $[4,7,11,14,18,19,24]$ and references given there. The idea of employing the method to prove generation theorems for semigroups of operators goes apparently back to W. Feller who constructed the semigroup of the minimal (and the reflected) Brownian motion on $\mathbb{R}^{+}$by noting that the space of odd (even) functions is left invariant by the semigroup of the unrestricted Brownian motion on $\mathbb{R}$, see [12], pp. 341-343, comp. [3]. These semigroups are generated by the one-dimensional Laplacian with Dirichlet and Neumann boundary conditions at the origin, respectively. A question whether a similar construction can be carried out also in the case of more general boundary conditions, seems to have been opened for

Communicated by Jerome A. Goldstein.

\footnotetext{
A. Bobrowski $(\bowtie)$

Department of Mathematics, Faculty of Electrical Engineering and Computer Science,

Lublin University of Technology, Nadbystrzycka 38A, 20-618 Lublin, Poland

e-mail: a.bobrowski@pollub.pl
} 
decades. The method of images hinges on constructing an extension of a function $f \in C[0, \infty]$ to the whole of $\mathbb{R}$ in such a way that the subspace of extensions of all $f \in C[0, \infty]$ is invariant for the unrestricted Brownian motion semigroup, but it has been unclear how this extension should be derived from the given boundary condition. Recently, in [6] the problem was implicitly solved in the case of Robin boundary condition, corresponding to the elastic Brownian motion [16, 20]. In [2], we show how to construct such extensions for general boundary conditions discussed in [20]. This note is devoted to further examples of an application of the method in the theory of semigroups of operators. These include a more direct semigroup-theoretic treatment of abstract delay differential equations [9], a simplified derivation of the form of the McKendrick semigroup [26], and the generation theorem for a semigroup describing kinase activity in the recent model of Kaźmierczak and Lipniacki [17]. For an alternative way of dealing with boundary conditions in semigroup theory see [13].

A proper context for the method of images in the theory of semigroups is provided by the notion of a similar semigroup [9] (or: isomorphic semigroup [3]). To recall, given two strongly continuous semigroups $\left\{\mathrm{e}^{t A}, t \geq 0\right\}$ and $\left\{\mathrm{e}^{t G}, t \geq 0\right\}$ in Banach spaces $\mathbb{X}$ and $\mathbb{B}$, respectively, we say that they are similar or isomorphic if there exists an isomorphism $I: \mathbb{X} \rightarrow \mathbb{B}$ such that $\mathrm{e}^{t A}=I^{-1} \mathrm{e}^{t G} I, t \geq 0$. Then, the generators $A$ and $G$ are related by

$$
D(A)=\{x \in \mathbb{X} ; I x \in D(G)\}, \quad A x=I^{-1} G I x .
$$

A particular case of this situation is as follows. Suppose that $\mathbb{Y}$ is a Banach space, $\Lambda$ is a subset of $\mathbb{R}, \mathbb{X}$ is a space of $\mathbb{Y}$-valued functions on $\Lambda$, and we are interested in proving that a certain operator $A$ in $\mathbb{X}$ is the generator of a strongly continuous semigroup. Assume also that there exists $\Lambda \subset \Lambda^{\prime} \subset \mathbb{R}$ and a strongly continuous semigroup $\{T(t), t \geq 0\}$ of operators in a space $\mathbb{B}_{0}$ of $\mathbb{Y}$-valued functions on $\Lambda^{\prime}$, generated by an operator $G_{0}$ resembling $A$. Usually, $A$ is " $G_{0}$ with a boundary condition". One way to approach such a problem is extending functions in $\mathbb{X}$ to functions in $\mathbb{B}_{0}$ so that the set of these extensions is an invariant subspace $\mathbb{B} \subset \mathbb{B}_{0}$ for $\{T(t), t \geq 0\}$. Then, the part $G$ of $G_{0}$ in $\mathbb{B}$ is the generator of $\left\{T(t)_{\mid \mathbb{B}}, t \geq 0\right\}$. Moreover, quite often, $\mathbb{X}$ and $\mathbb{B}$ are then isomorphic with natural isomorphism $I$ mapping a function on $\Lambda$ to its extension to $\Lambda^{\prime}$, and $R:=I^{-1}$ mapping a function on $\Lambda^{\prime}$ to its restriction on $\Lambda$. Then, there exists the semigroup in $\mathbb{X}$ that is similar to $\left\{\mathrm{e}^{t G}, t \geq 0\right\}$. Hence, to show that $A$ is the generator, it suffices to show (1.1). Then, as a bonus, we obtain the explicit form of $\mathrm{e}^{t A}$, referred to as the abstract Kelvin formula:

$$
\mathrm{e}^{t A} f=R \mathrm{e}^{t G} I f, \quad f \in \mathbb{X}, t \geq 0 .
$$

The non-trivial part in the procedure described above is finding the way of extending functions on $\Lambda$ to functions on $\Lambda^{\prime}$ so that all the remaining steps are valid. To explain the idea of deriving formulae for such extensions, let us recall that any strongly continuous semigroup leaves the domain of its generator invariant. Hence, if members $f$ of $D(A)$ are characterized by a functional equation (a boundary condition), say $F(f)=0$, then we must have $F\left(\operatorname{Re}^{t G} I f\right)=0, t \geq 0$. This, when coupled with $(I f)_{\mid \Lambda}=f$, often determines $I f, f \in D(A)$, and, by density of $D(A)$, all $I f$; see [2] and the examples that follow. As we will see, the method of images often leads to the most natural approach to the generation problem. 


\section{Delay differential equations}

The monograph [9], pp. 420-422 presents the following construction of a fundamental semigroup related to an abstract delay differential equation. Let $B$ be the generator of a semigroup $\left\{\mathrm{e}^{t B}, t \geq 0\right\}$ in a Banach space $\mathbb{Y}$ and, for a given $r>0$, let $\mathbb{X}=C([-r, 0], \mathbb{Y})$. Also, let $\Phi \in \mathcal{L}(\mathbb{X}, \mathbb{Y})$ be an abstract delay operator. The key Theorem 6.1 in [9], states that the operator $A f=f^{\prime}$ with domain $\mathcal{D}(A)=\{f \in$ $\left.C^{1}([-r, 0], \mathbb{Y}) ; f(0) \in D(B), f^{\prime}(0)=B[f(0)]+\Phi f\right\}$ is the generator of a strongly continuous semigroup in $\mathbb{X}$. The proof is carried in two steps. In the first step, it is shown that for $\Phi=0$, the semigroup generated by $A$ is given by the formula:

$$
\left(\mathrm{e}^{t A} f\right)(s)= \begin{cases}f(t+s), & t+s \leq 0, \\ \mathrm{e}^{(t+s) B}[f(0)], & t+s \geq 0 .\end{cases}
$$

Then, it is shown that the general case may be obtained by the Desch-Schappacher perturbation theorem.

We will prove that the method of images allows obtaining this result directly. To this end, for $\omega \geq 0$, let $\mathbb{X}_{\omega}$ be the space of continuous $\mathbb{Y}$-valued functions $g$ on $[-r, \infty)$ such that $\||g|\|:=\sup _{t \geq-r} \mathrm{e}^{-\omega t}\|g(t)\|<\infty$. $\left(\mathbb{X}_{\omega},\||\cdot|\|\right)$ is a Banach space. In the procedure described in Sect. 1, this space will play the role of $\mathbb{B}_{0}$ (for large $\omega$ ). The formula

$$
T(t) g(s)=g(s+t),
$$

defines a strongly continuous semigroup $T$ in $\mathbb{X}_{\omega}$. Its infinitesimal generator is $G_{0} g=g^{\prime}$ with domain composed of differentiable $g \in \mathbb{X}_{\omega}$ with $g^{\prime} \in \mathbb{X}_{\omega}$.

As explained in Sect. 1, if the abstract Kelvin formula (1.2) is to hold, the extension $g:=I f$ of $f \in D(A)$ must be chosen so that $R T(t) g \in D(A), t \geq 0$. In other words, $g$ must be differentiable with

$$
g^{\prime}(t)=B[g(t)]+\Phi R T(t) g, \quad t \geq 0 .
$$

This suggests that $g:=I f, f \in \mathbb{X}$ be defined as a mild solution to (2.2): a continuous function $g$ will be termed the $\Phi$-extension of $f \in \mathbb{X}$ iff

$$
R g=f \quad \text { and } \quad g(t)=\mathrm{e}^{t B}[f(0)]+\int_{0}^{t} \mathrm{e}^{(t-s) B} \Phi R T(s) g \mathrm{~d} s, \quad t \geq 0 .
$$

Certainly, (2.2) is the very delay differential equation we want to deal with via semigroup generated by $A$. Interestingly, but not surprisingly, the method of images recovers (2.2) from the boundary condition characterizing $D(A)$.

The appropriate existence and uniqueness result for $\Phi$-extensions is contained in the following lemma.

\section{Lemma 2.1}

(a) For $f \in \mathbb{X}$, the $\Phi$-extension exists and is unique.

(b) If $g$ is the $\Phi$-extension of $f \in \mathbb{X}$, then $T(t) g$ is the $\Phi$-extension of $R T(t) g, t \geq 0$.

(c) There is $\omega_{0} \geq 0$ such that for all $\omega \geq \omega_{0}, g \in \mathbb{X}_{\omega}$ for all $f \in \mathbb{X}$. 
Proof Without loss of generality, we assume $M:=\sup _{t \geq 0}\left\|\mathrm{e}^{t B}\right\|<\infty$.

(a) Let $\Delta=\frac{3}{4 M\|\Phi\|}$ and for fixed $f \in \mathbb{X}$, let $\mathbb{Z}$ be the complete metric space of functions $g$ in $C([-r, \Delta], \mathbb{Y})$ such that $R g=f$; the metric here is $d\left(g_{1}, g_{2}\right)=$ $\sup _{t \in[0, \Delta]}\left\|g_{1}(t)-g_{2}(t)\right\|$. Let us consider $U: \mathbb{Z} \rightarrow \mathbb{Z}$ given by $U g(t)=\mathrm{e}^{t B}[f(0)]+$ $\int_{0}^{t} \mathrm{e}^{(t-s) B} \Phi R T(s) g \mathrm{~d} s$. (For $s \in[0, \Delta], T(s) g(u), u \in[-r, 0]$ is well-defined.) We have

$$
d\left(U g_{1}, U g_{2}\right)=\sup _{t \in[0, \Delta]}\left\|\int_{0}^{t} \mathrm{e}^{(t-s) B} \Phi R T(s)\left[g_{1}-g_{2}\right] \mathrm{d} s \leq \Delta\right\| \Phi \| M \leq \frac{3}{4} d\left(g_{1}, g_{2}\right) .
$$

Hence, by the Banach fixed point theorem [8], there exists a unique fixed point $g$ of $U$, solving (2.3) for $t \in[0, \Delta]$. An analogous argument shows that if the extension $g$ is defined on $[-r, n \Delta]$, then it may be continued to $[-r,(n+1) \Delta]$. Hence, $g$ may be defined in a unique way for all $t \geq 0$.

(b) We check that

$$
g(t+u)=\mathrm{e}^{u B}[g(t)]+\int_{0}^{u} \mathrm{e}^{(u-s) B} \Phi R T(s)[T(t) g] \mathrm{d} s, \quad u, t \geq 0 .
$$

By uniqueness of $\Phi$-extensions, this shows (b).

(c) By (b), the map $f \mapsto R T(t) g$ is a strongly continuous semigroup in $\mathbb{X}$. Hence, there exists $\omega \geq 0$ and $M_{1} \geq 1$ such that $\|g(t)\|_{\mathbb{Y}} \leq\|R T(t) g\|_{\mathbb{X}} \leq M_{1} \mathrm{e}^{\omega t}\|f\|_{\mathbb{X}}, t \geq 0$, $f \in \mathbb{X}$. This implies $g \in \mathbb{X}_{\omega}$.

Theorem 2.2 A is the generator of a strongly continuous semigroup in $\mathbb{X}$. Moreover, the abstract Kelvin formula

$$
\mathrm{e}^{t A} f(s)=g(s+t), \quad f \in \mathbb{X}, t \geq 0, s \in[-r, 0]
$$

holds with $g$ being the $\Phi$-extension of $f$.

Proof Let $\omega$ be as in Lemma 2.1. By the Closed Graph Theorem, $I$ mapping $f \in \mathbb{X}$ to its $\Phi$-extension is continuous. Since $I$ has a continuous inverse $R$, the range $\mathbb{B}$ of $I$ is a closed linear subspace of $\mathbb{X}_{\omega}$. By Lemma 2.1(b), $\mathbb{B}$ is invariant for $T$.

We need to show that (1.1) holds with $G$ being the part of $G_{0}$ in $\mathbb{B}$. It suffices to show that $D(A)$ is the set of functions $f \in \mathbb{X}$ whose $\Phi$-extensions $g$ are differentiable with $g^{\prime} \in \mathbb{B}$. If such an extension exists, by (2.3), $f(0) \in D(B)$, the right-hand derivative $g_{+}^{\prime}(0)$ of $g$ at 0 equals $B[f(0)]+\Phi f$, and $f$ is continuously differentiable in $t \in[-r, 0]$ with $f^{\prime}(0)=g_{+}^{\prime}(0)=B[f(0)]+\Phi f$, proving that $f \in D(A)$. Conversely, let $f \in D(A)$ and, given $\lambda>\omega$, let $h:=\lambda f-f^{\prime} \in \mathbb{X}$. Since $\left\|\mathrm{e}^{t G}\right\| \leq \mathrm{e}^{\omega t}$, there exists $g \in D(G)$ such that $R(\lambda g-G g)=h$. Thus, $\lambda f(s)-f^{\prime}(s)=\lambda g(s)-g^{\prime}(s)$, $s \in[-r, 0]$, implying $R g=f$. By the uniqueness of $\Phi$-extensions, we are done.

Remark 2.3 For $\Phi=0$, the $\Phi$-extensions satisfy $g(t)=\mathrm{e}^{t B}[f(0)], t \geq 0$. Hence, (2.1) is a particular case of (2.5). Moreover, with (2.5) at hand, Lemmas 6.2 and 6.5 in [9] are immediate, and the whole analysis presented in Sects. VI 6, a-b of the monograph greatly simplifies. 


\section{The McKendrick semigroup}

Let $L^{1}\left(\mathbb{R}^{+}\right)$be the space of Lebesgue integrable functions on $\mathbb{R}^{+}$with the norm $\|\phi\|_{L^{1}\left(\mathbb{R}^{+}\right)}=\int_{\mathbb{R}^{+}}|\phi(a)| \mathrm{d} a$ and let $\mathrm{e}_{\omega}(a)=\mathrm{e}^{-\omega a}$ (this function will be treated both as a function on $\mathbb{R}$ and as its restriction to $\mathbb{R}^{+}$). Furthermore, let $L_{\omega}^{1}\left(\mathbb{R}^{+}\right)$be the space of (classes of) measurable functions $\phi$ on $\mathbb{R}^{+}$such that $\mathrm{e}_{\omega} \phi \in L^{1}\left(\mathbb{R}^{+}\right)$with the norm $\|\phi\|_{L_{\omega}^{1}\left(\mathbb{R}^{+}\right)}=\left\|\mathrm{e}_{\omega} \phi\right\|_{L^{1}\left(\mathbb{R}^{+}\right)}$, and $L_{\omega}^{1}(\mathbb{R})$ be the space of (classes of) measurable functions $\varphi$ on $\mathbb{R}$ such that $\|\varphi\|_{L_{\omega}^{1}(\mathbb{R})}:=\int_{\mathbb{R}}\left(\mathrm{e}^{\omega a} \wedge 1\right)|\varphi(a)| \mathrm{d} a<\infty$. We note that $L_{\omega}^{1}(\mathbb{R})$ is isometrically isomorphic to $L^{1}\left(\mathbb{R}^{+}\right) \times L_{\omega}^{1}\left(\mathbb{R}^{+}\right)$for the norm $\|(\phi, \psi)\|_{L^{1}\left(\mathbb{R}^{+}\right) \times L_{\omega}^{1}\left(\mathbb{R}^{+}\right)}=\|\phi\|_{L^{1}\left(\mathbb{R}^{+}\right)}+\|\psi\|_{L_{\omega}^{1}\left(\mathbb{R}^{+}\right)}$. The isomorphism is given by $J \varphi=(\phi, \psi), \phi \in L_{\omega}^{1}(\mathbb{R})$ where $\phi(a)=\varphi(a)$ and $\psi(a)=\varphi(-a), a \geq 0$.

Let $b$ and $\mu$ be non-negative, bounded, measurable functions on $\mathbb{R}^{+}$; equivalently, we treat $b$ and $\mu$ as functions on $\mathbb{R}$ that vanish on the negative half-axis. Let $A$ be an operator in $L^{1}\left(\mathbb{R}^{+}\right)$given by $A \phi=-\phi^{\prime}-\mu \phi$ with domain $\operatorname{Ker} F$ where $F$ : $W^{1,1}\left(\mathbb{R}^{+}\right) \rightarrow \mathbb{R}$ is given by

$$
F(\phi)=\phi(0)-\int_{\mathbb{R}^{+}} b(a) \phi(a) \mathrm{d} a
$$

and $W^{1,1}$ is the Sobolev space of integrable, absolutely continuous functions with derivatives in $L^{1}\left(\mathbb{R}^{+}\right)$. The abstract Cauchy problem related to $A$ is called the McKendrick equation [22] or Sharpe-Lotka-McKendrick equation [25, 26], or LotkaMcKendrick equation [15]. In the population dynamics interpretation of this problem, $\phi$ is an age-profile of an age-structured population with births and deaths governed by $b$ and $\mu$, respectively. For this reason, it is customary to write the argument of $\phi$ as $a$, ' $a$ ' standing for 'age'.

To show that $A$ generates a strongly continuous semigroup in $L^{1}\left(\mathbb{R}^{+}\right)$we consider the operator $G_{0}$ in $L_{\omega}^{1}(\mathbb{R})$ defined by

$$
D\left(G_{0}\right)=W_{\omega}^{1,1}(\mathbb{R}), \quad G_{0} \phi=-\phi-\mu \phi,
$$

where $W_{\omega}^{1,1}$ is the Sobolev-type space of absolutely continuous members of $L_{\omega}^{1}(\mathbb{R})$ with derivatives in $L_{\omega}^{1}(\mathbb{R})$; recall that $\mu(a)=0$ for $a<0 . G_{0}$ generates the strongly continuous semigroup in $L_{\omega}^{1}(\mathbb{R})$ given by

$$
T(t) \phi(a)=\mathrm{e}^{-\int_{a-t}^{a} \mu(r) \mathrm{d} r} \phi(a-t),
$$

and we have $\|T(t)\|_{\mathcal{L}\left(L_{\omega}^{1}(\mathbb{R})\right)} \leq \mathrm{e}^{\omega t}, t \geq 0$.

We proceed as in Sect. 1, to find an extension $\varphi \in L_{\omega}^{1}(\mathbb{R})$ of $\phi \in L^{1}\left(\mathbb{R}^{+}\right)$. To this end, it suffices to find $\psi \in L_{\omega}^{1}\left(\mathbb{R}^{+}\right)$related to $\varphi$ via $\psi(t)=\varphi(-t), t \geq 0$. For $\phi \in D(A)$, we must have $F(T(t) \varphi)=0$, or $\varphi(-t)=\int_{0}^{\infty} \mathrm{e}^{-\int_{a-t}^{a} \mu(r) \mathrm{d} r} \varphi(a-t)$ $b(a) \mathrm{d} a, t \geq 0$. Hence, $\psi$ must satisfy

$$
\psi=\psi * b_{\mu}+C \phi
$$

where $C \phi(t):=\int_{t}^{\infty} \mathrm{e}^{-\int_{a-t}^{a} \mu(r) \mathrm{d} r} \phi(a-t) b(a) \mathrm{d} a, b_{\mu}(a)=\mathrm{e}^{-\int_{0}^{a} \mu(r) \mathrm{d} r} b(a), a \geq 0$ and $*$ denotes convolution. Note that $b_{\mu}$ is bounded, $b$ being bounded. 


\section{Lemma 3.1}

(a) For $\phi \in L^{1}\left(\mathbb{R}^{+}\right),\|C \phi\|_{L_{\omega}^{1}\left(\mathbb{R}^{+}\right)} \leq\|\phi\|_{L^{1}\left(\mathbb{R}^{+}\right)} \frac{\|b\|_{\infty}}{\omega}$.

(b) Let $\omega>\left\|b_{\mu}\right\|_{\infty}:=\sup _{a \geq 0}\left|b_{\mu}(a)\right|$. For $\phi \in L^{1}\left(\mathbb{R}^{+}\right)$, there exist unique $\psi \in$ $L_{\omega}^{1}\left(\mathbb{R}^{+}\right)$solving (3.2).

(c) For $\phi \in \operatorname{Ker} F$ there exists unique $\varphi \in L_{\omega}^{1}(\mathbb{R})$ such that for all $t \geq 0, T(t) \varphi \in$ $\operatorname{Ker} F$ and $\varphi_{\mathbb{R}^{+}}=\phi$. Here, we treat $F$ as a functional on $W_{\omega}^{1,1}(\mathbb{R})$; in particular $T(t) \varphi \in W_{\omega}^{1,1}(\mathbb{R})$.

Proof (a) Follows by a straightforward calculation. In particular $C$ is a bounded operator $C: L^{1}\left(\mathbb{R}^{+}\right) \rightarrow L_{\omega}^{1}\left(\mathbb{R}^{+}\right)$.

(b) The norm of the map $L_{\omega}^{1}\left(\mathbb{R}^{+}\right) \ni \psi \mapsto \psi * b_{\mu} \in L_{\omega}^{1}\left(\mathbb{R}^{+}\right)$does not exceed $\frac{\left\|b_{\mu}\right\|_{\infty}}{\omega}<1$. Hence, the only solution to (3.2) is $\psi=\sum_{n \geq 0} b_{\mu}^{n *} * C \phi$.

(c) If $\phi$ is absolutely continuous, so is $C \phi$ with $(C \phi)^{\prime}=-\phi(0) b_{\mu}-\kappa$ where $\kappa(t)=\int_{0}^{\infty}\left[\mu(a) \phi(a)+\phi^{\prime}(a)\right] \mathrm{e}^{-\int_{a}^{a+t} \mu(r) \mathrm{d} r} b(a+t) \mathrm{d} a$. Since $b_{\mu}$ and $\kappa$ are bounded $(C \phi)^{\prime}$ belongs to $L_{\omega}^{1}\left(\mathbb{R}^{+}\right)$. Therefore, $\psi$ is absolutely continuous with $\psi^{\prime}$ equal to $\sum_{n \geq 0} b_{\mu}^{n *} *(C \phi)^{\prime}+\phi(0) \sum_{n \geq 0} b_{\mu}^{n *} \in L_{\omega}^{1}\left(\mathbb{R}^{+}\right)$, because $\sum_{n \geq 0} b_{\mu}^{n *}$ converges in $L_{\omega}^{1}\left(\mathbb{R}^{+}\right)$. Since for $\phi \in \operatorname{Ker} F, \phi(0)=\int_{\mathbb{R}^{+}} \phi(a) b(a) \mathrm{d} a=\psi(0)$, we have $\varphi \in$ $W_{\omega}^{1,1}(\mathbb{R})$, and (3.2) forces $T(t) \varphi \in \operatorname{Ker} F$.

For $\phi \in L^{1}\left(\mathbb{R}^{+}\right)$, the $\varphi \in L_{\omega}^{1}(\mathbb{R})$ defined by $\varphi(a)=\phi(a), \varphi(-a)=\psi(a), a \geq 0$ where $\psi$ solves (3.2), will be referred to as the $(b, \mu)$-extension of $\phi$.

Theorem 3.2 $A$ is the generator of a strongly continuous semigroup in $L^{1}\left(\mathbb{R}^{+}\right)$. Furthermore,

$$
\mathrm{e}^{t A} \phi(a)=T(t) \varphi(a)=\mathrm{e}^{-\int_{a-t}^{a} \mu(r) \mathrm{d} r} \varphi(a-t), \quad a, t \geq 0,
$$

where $\varphi$ is the $(b, \mu)$-extension of $\phi$.

Proof Let $\mathbb{B}$ be the space of $(b, \mu)$-extensions of members of $L^{1}\left(\mathbb{R}^{+}\right)$. By Lemma 3.1 (b), $\mathbb{B} \subset L_{\omega}^{1}(\mathbb{R})$ for sufficiently large $\omega$. As in Theorem 2.2, we show that $\mathbb{B}$ is closed. Fix $s \geq 0$ and $\phi \in D(A)=\operatorname{Ker} F$. By Lemma 3.1 (c), $T(t+s) \varphi \in \operatorname{Ker} F, t \geq 0$. Thus, by uniqueness of $(b, \mu)$-extensions $T(s) \varphi$ is the $(b, \mu)$-extension of $[T(s) \varphi]_{\mathbb{R}^{+}} \in$ $L^{1}\left(\mathbb{R}^{+}\right)$. Hence, the image of $D(A)=\operatorname{Ker} F$ is invariant for $T$ and so is $\mathbb{B}$, the image of $D(A)$ being dense in $\mathbb{B}$.

Let $G$ be the part of $G_{0}$ in $\mathbb{B}$ and let $\varphi \in D(G)$. Then, $\phi:=\varphi_{\mathbb{R}^{+}} \in L^{1}\left(\mathbb{R}^{+}\right)$is absolutely continuous with $\phi^{\prime} \in L^{1}\left(\mathbb{R}^{+}\right)$and, by $(3.2), \phi(0)=\psi(0)=\int_{\mathbb{R}^{+}} \phi(a) b(a) \mathrm{d} a$, proving that $\phi \in D(A)$. Conversely, if $\phi \in D(A)$, then, by Lemma 3.1 (c), its $(b, \mu)$ extension belongs to $W_{\omega}^{1,1}(\mathbb{R})$. This shows $(1.1)$.

Remark 3.3 A semigroup-theoretical treatment of the McKendrick equation usually involves some kind of perturbation argument, see [5], pp. 241-244 or [10], pp. 216-219. Our method and formula (3.3) are worth comparing with the remark on the bottom of the page 244 in [5], pp. 8-12 and formula (3.3) in [15], or pp. 138-151 
in [21]. In fact, a natural, intuitive way of solving the McKendrick equation is by solving a renewal equation for the total birth rate, which is equivalent to (3.2). Interestingly, the method of images leads to (3.2) without appealing to population dynamics intuitions.

\section{A Bessel process with mass inflow}

Let $\mathbb{X}=C[0,1]$ be the space of real continuous functions on $[0,1]$ and let $h(x):=$ $x, x \in[0,1]$. Let $D(A)$ be composed of functions $f$ such that (a) $h f$ is twice continuously differentiable for $x \in(0,1]$ and $\frac{1}{2 h}(h f)^{\prime \prime}$ may be extended to a continuous function on $[0,1]$, and (b) $f^{\prime}(1)=\mu f(1)$, where $\mu>0$ is a given constant. We will show that $A$ given by $A f=\frac{1}{2 h}(h f)^{\prime \prime}$ is the generator of a semigroup in $\mathbb{X}$.

The operator $A$ is clearly akin to $G_{0}$ in $C[0, \infty]$ given by $G_{0} f=\frac{1}{2 h}(h f)^{\prime \prime}$ with domain composed of those $f \in C[0, \infty]$ for which $h f$ is twice continuously differentiable with $\frac{1}{2 h}(h f)^{\prime \prime}$ extendable to a function in $C[0, \infty]$; here $h(x)=x, x \in[0, \infty)$. $G_{0}$ generates the strongly continuous semigroup in $C[0, \infty]$ given by

$$
T(t) f(x)=\left\{\begin{array}{ll}
\int_{0}^{\infty} \frac{y}{x}\left[q_{t}(x-y)-q_{t}(x+y)\right] f(y) \mathrm{d} y, & x>0, \\
\frac{2}{t} \int_{0}^{\infty} q_{t}(y) y^{2} f(y) \mathrm{d} y, & x=0,
\end{array} \quad t>0,\right.
$$

where $q_{t}(x)=\frac{1}{\sqrt{2 \pi t}} \mathrm{e}^{-\frac{x^{2}}{2 t}}$. To show this, we note that the Laplace transform of the right-hand side of (4.1) equals, for $x>0, \lambda>0$,

$$
g(x):=\left(\lambda-G_{0}^{*}\right)^{-1} f(x)=\frac{1}{x \sqrt{2 \lambda}} \int_{0}^{\infty}\left[\mathrm{e}^{-\sqrt{2 \lambda}|x-y|}-\mathrm{e}^{-\sqrt{2 \lambda}(x+y)}\right] y f(y) \mathrm{d} y,
$$

where, for the sake of the present argument, $G_{0}^{*}$ denotes the generator of (4.1). Hence, a direct computation shows that $g \in D\left(G_{0}\right)$ and $\lambda g-G_{0} g=f$, implying $G_{0} g=G_{0}^{*} g$. Since $\lambda-G_{0}^{*}$ is injective with range equal to $\mathbb{X}, G_{0}$ cannot be a proper extension of $G_{0}$, proving the claim.

$T$ given by (4.1) is the semigroup of the three dimensional Bessel process, a process whose realization is the modulus of the three dimensional Brownian motion. In other words,

$$
T(t) f(x)=E f(|x+w(t)|)
$$

where $w(t), t \geq 0$ is a standard three-dimensional Brownian motion, $x \in \mathbb{R}$ is identified with $(x, 0,0) \in \mathbb{R}^{3}$ and $E$ stands for expectation, see [23], p. 251.

The operator $A_{1}$ given by $A_{1} f=A f-f, f \in C[0,1]$ appears implicitly in the recent model of kinase activity in a living cell [17]. In this model, the cell is viewed as a unit ball where the active kinase particles diffuse freely and may be randomly inactivated (hence the term $-f$ in the definition of $A_{1} f$ ). Since it is assumed that the active kinase concentration depends merely on the distance from the ball center, we are actually dealing with a 3 dimensional Bessel process (with killing). The inactive kinase particles perform a similar motion but, upon touching the boundary some of them are being activated. The boundary condition $f^{\prime}(1)=\mu f(1)$ describes 
the "inflow" [17] of activated kinase particles reflected from the boundary $x=1$. For simplicity, we do not take into account the fact that the number of kinase particles (active and inactive) is limited. Neither do we take into account fluctuations of activity of surface receptors; the detailed semigroup-theoretic treatment of the complete model exceeds the scope of this article, and will be the subject of further research.

Let $\mathbb{X}_{\omega}, \omega>0$ be the space of continuous functions $f$ such that $\mathrm{e}_{\omega} f \in C[0, \infty]$ (where $\mathrm{e}_{\omega}(x)=\mathrm{e}^{-\omega x}, x \geq 0$ ). For the norm $\|f\|_{\omega}=\left\|\mathrm{e}_{\omega} f\right\|_{C[0, \infty]}, \mathbb{X}_{\omega}$ is a Banach space. Our main result is:

Theorem 4.1 There is $\omega>0$ such that for each $f \in \mathbb{X}$ there exists $g_{\mu}: \mathbb{R}^{+} \rightarrow \mathbb{R}$ such that $\left(g_{\mu}\right)_{\mid[0,1]}=f, h g_{\mu} \in \mathbb{X}_{\omega}$ and

$$
S(t) f(x)=\left\{\begin{array}{ll}
\int_{0}^{\infty} \frac{y}{x}\left[q_{t}(x-y)-q_{t}(x+y)\right] g_{\mu}(y) \mathrm{d} y, & x>0, \\
\frac{2}{t} \int_{0}^{\infty} q_{t}(y) y^{2} g_{\mu}(y) \mathrm{d} y, & x=0,
\end{array} \quad t>0,\right.
$$

is a strongly continuous semigroup in $\mathbb{X}$ with generator $A$.

Formula (4.3) may be equivalently expressed as (compare (4.2))

$$
S(t) f(x)=E g_{\mu}(|x+w(t)|), \quad x \in[0,1] .
$$

Because of the relative complexity of (4.3), it is difficult to derive $g_{\mu}$ directly. However, in (4.9) and (4.10) we will express $S$ in terms of the much simpler, canonical cosine family

$$
C(t) f(x)=\frac{1}{2}[f(x+t)+f(x-t)], \quad x, t \in \mathbb{R},
$$

in $\mathbb{Z}_{\omega}$, the space of continuous functions $f$ on $\mathbb{R}$ such that both $f_{\mid \mathbb{R}^{+}}$and $\mathbb{R}^{+} \ni$ $x \mapsto f(-x)$ belong to $\mathbb{X}_{\omega}$; the norm in $\mathbb{Z}_{\omega}$ is $\|f\|=\sup _{x \in \mathbb{R}} \mathrm{e}^{-|x| \omega}|f(x)|$. Therefore, in Lemma 4.2, we define extensions of $f \in \mathbb{X}$ to $f_{v} \in \mathbb{Z}_{\omega}$ in such a way that the subspace of such extensions is invariant for $C$. Then, we define $g_{\mu}$ in terms of $f_{\nu}$.

Lemma 4.2 Let $v:=\mu+1$ and $F_{v}$ be the functional in $\mathbb{Z}_{\omega}$ given by $F(f)=v f(1)-$ $f^{\prime}(1)$, with domain $D(F)$ composed of continuously differentiable $f \in \mathbb{Z}_{\omega}$. There is $\omega>0$ such that for continuously differentiable $f \in C_{0}(0,1]$ with $f^{\prime}(1)=v f(1)$ there exists a unique $f_{v} \in \mathbb{Z}_{\omega}$ such that:

(a) $C(t) f_{v}$ is odd for $t \in \mathbb{R}$,

(b) $C(t) f_{v} \in \operatorname{Ker} F$ for $t \in \mathbb{R}$.

Proof Let $P \in \mathcal{L}\left(\mathbb{Z}_{\omega}\right)$ be given by $P f(x)=f(1-x), x \in \mathbb{R}$, and let $F_{P}:=F \circ P$, whence $F f=f^{\prime}(0)+v f(0), f \in D\left(F_{P}\right):=D(F)$. By a straightforward generalization of Lemma 1 in [2], (b) holds iff $f_{v, P}:=P f_{v}$ satisfies

$$
f_{v, P}(x)=f_{v, P}(-x)+2 v \int_{0}^{-x} \mathrm{e}^{v(-x-y)} f_{v, P}(y) \mathrm{d} y, \quad x \leq 0,
$$


and this is true iff $f_{v}$ satisfies

$$
f_{v}(x)=f_{v}(2-x)+2 v \int_{2-x}^{1} \mathrm{e}^{v(x+y-2)} f_{v}(y) \mathrm{d} y, \quad x \geq 1 .
$$

Similarly, (a) holds iff $f_{v}$ is odd. Therefore, we define $f_{v}$ by induction: having defined it on $[-n, n+1], n \geq 0$ we put

$$
f_{v}(x)= \begin{cases}f_{v}(-x), & x \in[-n-1,-n], \\ f_{v}(2-x)+2 v \int_{2-x}^{1} \mathrm{e}^{v(x+y-2)} f_{v}(y) \mathrm{d} y, & x \in[n+1, n+2] .\end{cases}
$$

(Compare pp. 117-119 in [6].) We note that these formulae are local in the sense that the definition of $f_{v}(x), x \in[-n-1,-n]$ depends merely on $f_{v}(x), x \in[n, n+1]$ and the definition of $f_{v}(x), x \in[n+1, n+2]$ depends merely on $f_{v}(x), x \in[-n, 1]$. Hence, $f_{v}$ is odd and (4.6) holds.

Formula (4.7) defines $f_{v}$ for all, not necessarily differentiable, $f \in C_{0}(0,1]$. This, not necessarily differentiable, $f_{v}$ will be referred to as the $(C, v)$-extension of $f$.

Fix $s \in \mathbb{R}$ and consider $C(s) f_{v}$ for a continuously differentiable $f$ such that $f^{\prime}(1)=v f(1)$. Then, by cosine functional equation, $C(t) C(s) f_{v}$ is odd and belongs to $\operatorname{Ker} F$. Hence, by uniqueness of $(C, v)$-extensions,

$$
C(s) f_{v} \text { is the }(C, v) \text {-extension of }\left[C(s) f_{v}\right]_{\mid[0,1]} .
$$

Since such functions $f$ are dense in $\mathbb{X}$, the same is true for all $f \in \mathbb{X}$. It follows that $C_{0}(t), t \in \mathbb{R}$ defined by $C_{0}(t) f=\left[C(t) f_{v}\right]_{\mid[0,1]}, f \in \mathbb{X}$ is a strongly continuous cosine family. Hence, there exist $M \geq 1, \omega_{0}>0$ such that $\left\|C_{0}(t)\right\|_{\mathcal{L}(\mathbb{X})} \leq M \mathrm{e}^{\omega_{0} t}$.

Fix $t \geq \frac{3}{2}, t=n+t^{\prime}$ where $t^{\prime} \in\left[\frac{1}{2}, \frac{3}{2}\right], n \geq 1$. Then $\left|f_{v}(t)-f_{v}(t-1)\right|=\mid f_{v}(1 / 2+$ $s)+f_{v}(1 / 2-s)\left|\leq 2 \sup _{x \in[0,1]}\right| C(s) f_{v}(x) \mid \leq 2 M \mathrm{e}^{\omega_{0}\left(t-\frac{1}{2}\right)}\|f\|_{\mathbb{X}}=K \mathrm{e}^{\omega_{0} t}$ where $s=$ $t-1 / 2$ and $K$ depends on $f$. Therefore,

$$
\begin{aligned}
\left|f_{v}(t)\right| & \leq\left|f_{v}(t-1)\right|+K \mathrm{e}^{\omega_{0} t} \leq\left|f_{v}(t-2)\right|+K \mathrm{e}^{\omega_{0}(t-1)}+K \mathrm{e}^{\omega_{0} t} \leq \cdots \\
& \leq\left|f_{v}\left(t^{\prime}\right)\right|+K \mathrm{e}^{\omega_{0} t}\left[\mathrm{e}^{-\omega_{0}(n-1)}+\cdots+1\right] \\
& \leq \sup _{t \in[1 / 2,3 / 2]}\left|f_{v}(t)\right|+\frac{K}{1-\mathrm{e}^{-\omega_{0}}} \mathrm{e}^{\omega_{0} t}, \quad t \geq 3 / 2 .
\end{aligned}
$$

This implies $f_{v} \in \mathbb{Z}_{\omega}$ for $\omega>\omega_{0}, f_{v}$ being odd.

Proof of Theorem 4.1 Step 1 . Let $\mathbb{Z}_{\omega, \mathrm{e}} \subset \mathbb{Z}_{\omega}$ be the subspace of $(C, v)$-extensions of elements of $\mathbb{X}$. Using the Closed Graph Theorem, we check that the map $\mathbb{X} \ni f \mapsto$ $f_{\nu} \in \mathbb{Z}_{\omega}$ is bounded. Hence, as before, we see that $\mathbb{Z}_{\omega, \mathrm{e}}$ is isomorphic to $\mathbb{X}$, and (4.8) shows that $\mathbb{Z}_{\omega, \mathrm{e}}$ is invariant for $C$. The generator $B$ of the canonical cosine family (4.5) restricted to $\mathbb{Z}_{\omega, \text { e }}$ is given by $B f=f^{\prime \prime}$ and its domain $D(B)$ is composed of twice continuously differentiable functions in $\mathbb{Z}_{\omega, \mathrm{e}}$ with second derivative in $\mathbb{Z}_{\omega, \mathrm{e}}$.

Let $B_{v}$ in $C_{0}(0,1]$ be given by $B_{v} f=f^{\prime \prime}$ with domain composed of twice continuously differentiable functions on $[0,1]$ such that $f^{\prime}(1)=v f(1)$. Using Lemma 4.2 
and arguing as in Theorem 2.2, we see that $D(B)$ is the set of $(C, v)$-extensions of elements of $D\left(B_{v}\right)$. Therefore, $B_{v}$ generates the cosine operator function in $\mathbb{X}$ given by

$$
C_{v}(t) f=C(t) f_{v} .
$$

Step 2. By the Weierstrass formula ([1], (3.102)), the semigroup generated by $\frac{1}{2} B_{v}$ is given by

$$
\begin{aligned}
\mathrm{e}^{\frac{1}{2} B_{v} t} f(x) & =\sqrt{\frac{2}{\pi t}} \int_{0}^{\infty} \mathrm{e}^{-\frac{s^{2}}{2 t} C(s) f_{v}(x) \mathrm{d} s} \\
& =\frac{1}{\sqrt{2 \pi t}} \int_{0}^{\infty}\left[\mathrm{e}^{-\frac{(s-x)^{2}}{2 t}}-\mathrm{e}^{-\frac{(s+x)^{2}}{2 t}}\right] f_{v}(s) \mathrm{d} s \quad x \geq 0,
\end{aligned}
$$

where we used the fact that $f_{v}$ is odd. Since for $f \in \mathbb{X}, h f \in C_{0}(0,1]$ we may define

$$
\begin{aligned}
S(t) f(x) & =\frac{1}{x} \mathrm{e}^{\frac{1}{2} B_{v} t}(h f)(x) \\
& =\int_{0}^{\infty} \frac{1}{x}\left[q_{t}(s-x)-q_{t}(s+x)\right](h f)_{\nu}(s) \mathrm{d} s, \quad x \in(0,1] .
\end{aligned}
$$

By Dominated Convergence Theorem, $S(t) f$ may be extended to a continuous function on $[0,1]$ by setting $S(t) f(0)=\frac{2}{t} \int_{0}^{\infty} y q_{t}(y)(h f)_{v}(y) \mathrm{d} y$. Then, (4.10) shows that $S$ is a semigroup of operators in $\mathbb{X}$. Using (4.4) and elementary properties of Brownian motion, we check that $S$ is strongly continuous. Setting $g_{\mu}(x)=$ $\frac{1}{x}(h f)_{v}(x), x>1$, gives (4.3).

Let $G$ be the generator of $S$. We are left with showing $G=A$. If $f \in D(G)$ and $G f=\lim _{t \rightarrow 0+} \frac{1}{t}(S(t) f-f)$, then $h G f=\lim _{t \rightarrow 0+} \frac{1}{t}\left[\mathrm{e}^{\frac{1}{2} B_{v} t}(h f)-h f\right]$. Hence, $h f$ belongs to $D\left(B_{v}\right)$ and $h G f=\frac{1}{2} B_{v}(h f)$. Therefore, $h f$ is twice continuously differentiable and $(h f)^{\prime}(1)=v(h f)(1)$. This implies $f \in D(A)$ and $G f=\frac{1}{2 h}(h f)^{\prime \prime}=A f$. Since $G$ is a generator, $A$ cannot be a proper extension of $G$, completing the proof.

Acknowledgement I would like to thank J. Banasiak, M. Lachowicz, D. Wrzosek and Z. Peradzyński for their remarks on the paper, and in particular for the remarks on the bibliography.

Open Access This article is distributed under the terms of the Creative Commons Attribution Noncommercial License which permits any noncommercial use, distribution, and reproduction in any medium, provided the original author(s) and source are credited.

\section{References}

1. Arendt, W., Batty, C.J.K., Hieber, M., Neubrander, F.: Vector-Valued Laplace Transforms and Cauchy Problems. Birkhäuser, Basel (2001)

2. Bobrowski, A.: Generation of cosine families via Lord Kelvin's method of images. J. Evol. Equ. (to appear). doi:10.1007/s00028-010-0065-Z

3. Bobrowski, A.: Functional Analysis for Probability and Stochastic Processes. Cambridge University Press, Cambridge (2005)

4. Bogdan, K., Żak, T.: On Kelvin transformation. J. Theor. Probab. 19(1), 89-120 (2006) 
5. Clément, Ph., Heijmans, H.J.A.M., Angenent, S., van Duijn, C.J., de Pagter, B.: One-parameter Semigroups. CWI Monographs, vol. 5. North-Holland, Amsterdam (1987)

6. Chill, R., Keyantuo, V., Warma, M.: Generation of cosine families on $L^{p}(0,1)$ by elliptic operators with Robin boundary conditions. In: Amann, H., et al. (ed.) Functional Analysis and Evolution Equations. The Günter Lumer Volume, pp. 113-130. Birkhäuser, Basel (2007)

7. Cheng, H.: On the method of images for systems of closely spaced conducting spheres. SIAM J. Appl. Math. 61(4), 1324-1337 (2000)

8. Edwards, R.E.: Functional Analysis. Theory and Applications. Holt, Rinehart and Winston, New York (1965). Republished by Dover, 1995

9. Engel, K.-J., Nagel, R.: One-parameter Semigroups for Linear Evolution Equations. Springer, Berlin (2000)

10. Engel, K.-J., Nagel, R.: Short Course on Operator Semigroups. Springer, Berlin (2006)

11. Fabrikant, V.I.: Generalized method of images in the crack analysis. Int. J. Eng. Sci. 35(12/13), 11591184 (1997)

12. Feller, W.: An Introduction to Probability Theory and Its Applications, vol. II, 2nd edn. Wiley, New York (1966), 1971

13. Greiner, G.: Perturbing the boundary conditions of a generator. Houst. J. Math. 13(2), 213-229 (1987)

14. Hobson, E.W.: Synthetical solutions in the conduction of heat. Proc. Lond. Math. Soc. 19, 279-299 (1887)

15. Iannelli, M.: Mathematical Theory of Age-Structured Population Dynamics. Applied Mathematical Monographs, vol. 7. Giardini Editori E Stampatori, Pisa (1995)

16. Ito, K., McKean, H.P. Jr.: Diffusion Processes and Their Sample Paths, Classics in Mathematics Series. Springer, Berlin (1996). Reprint of the 1974 edition

17. Kaźmierczak, B., Lipniacki, T.: Regulation of kinase activity by diffusion and feedback. J. Theor. Biol. 259, 291-296 (2009)

18. Körner, T.W.: Fourier Analysis. Cambridge University Press, Cambridge (1998)

19. Lindell, I.V., Wallén, K.H., Sihvola, A.H.: Electrostatic image theory for two intersecting conducting spheres. J. Electromagn. Waves Appl. 17(11), 1643-1660 (2003)

20. Mandl, P.: Analytical Treatment of One-Dimensional Markov Processes. Springer, New York (1968)

21. Metz, J.A.J., Diekman, O. (eds.): The Dynamics of Physiologically Structured Populations. Springer, Berlin (1986)

22. Murray, J.D.: Mathematical Biology, 2nd, edn. Springer, Berlin (1993)

23. Revuz, D., Yor, M.: Continuous Martingales and Brownian Motion, 3rd edn. Springer, Berlin (1999)

24. Volterra, V.: Note on the application of the method of images to problems of vibrations. Proc. Lond. Math. Soc. (2) 2(1), 327-331 (1905)

25. Webb, G.F.: Theory of Nonlinear Age-Dependent Population Dynamics. Dekker, New York (1985)

26. Webb, G.F.: Structured population dynamics. In: Rudnicki, R. (ed.) Mathematical Modelling of Population Dynamics. Banach Center Publications, vol. 63, pp. 123-163. IMPAN, Warszawa (2004) 